\title{
Elimination of flux-transformer crosstalk in multichannel SQUID magnetometers
}

\author{
H.J.M. ter Brake, F.H. Fleuren, J.A. Ulfman and J. Flokstra
}

Twente University of Technology, Department of Applied Physics, PO Box 217, 7500 AE

Enschede, The Netherlands

Received 1 April 1986; revised 11 September 1986

\begin{abstract}
Multichannel SQUID magnetometers are being developed for signal-field mapping in biomagnetic experiments. A problem that becomes more serious as the number of channels is increased is the crosstalk caused by the mutual inductances between the individual sensing coils. A simple and effective method for eliminating this crosstalk is presented in this Paper. The method is based on a rearrangement of the feedback loops which causes the flux-transformer circuits to become currentless. The feasibility of the method is verified experimentally.
\end{abstract}

Keywords: SQUIDs; magnetic fields; magnetometers

Today biomagnetic experiments require multichannel SQUID magnetometers to perform rapid signal-field mapping ${ }^{1}$. In the future, it is expected that magnetometers will have so many channels that a complete map will be obtained instantaneously. However, crosstalk between these channels may be a problem.

Crosstalk is caused by the mutual inductances between the individual sensing coils ${ }^{2}$; any signal current flowing in a sensing coil will induce error currents in the others. We define the crosstalk between identical coils as the ratio of the error current to the signal current, assuming all coils carry the same signal current. However, it is clear that the signal and crosstalk currents generally depend on the actual signal-field distribution. An estimation of crosstalk signals can be obtained by considering two second order gradiometers (diameter $20 \mathrm{~mm}$, baseline length $40 \mathrm{~mm}$ ) placed parallel to one another with a minimum distance of $1 \mathrm{~mm}$ between them. The crosstalk between these sensing coils is $\approx 5 \%$. When these coils are part of a multichannel system the crosstalk is even larger. In a hexagonal configuration a coil can have six neighbours, resulting in a crosstalk of $\approx 30 \%$.

The crosstalk can, of course, be minimized by separating the sensing coils and thus reducing the mutual inductances between them. However, this implies an important loss of information and it is, therefore, an impractical solution. Ilmoniemi et al. ${ }^{3}$ attempted to minimize the mutual inductances in another way. The compensating coils of the first order gradiometers in their four-channel system were constructed in such a way that the mutual inductances should vanish ${ }^{3}$. This method, however, can only be applied in very special sensing coil configurations: 1 , the coil sections of the gradiometers must not be coaxial; and, 2 , the mutual inductances for all sensing coils must be equivalent. For example, this method cannot be used in a hexagonal configuration of seven coils. Furthermore, even when the method is applicable it appears that a complete elimination of the crosstalk would be difficult, if not impossible, to realize ${ }^{3}$.
An alternative method, without these disadvantages, is presented in this Paper. It is shown that the crosstalk problem can be solved by rearranging the feedback loops of the SQUID systems in such a way that the fluxtransformer circuits become currentless. Experimental results are presented below.

\section{Theory}

A flux-transformer circuit containing a sensing coil is inductively coupled to a SQUID which, in turn, is coupled to the electronics, as shown in Figure 1 . The output of the

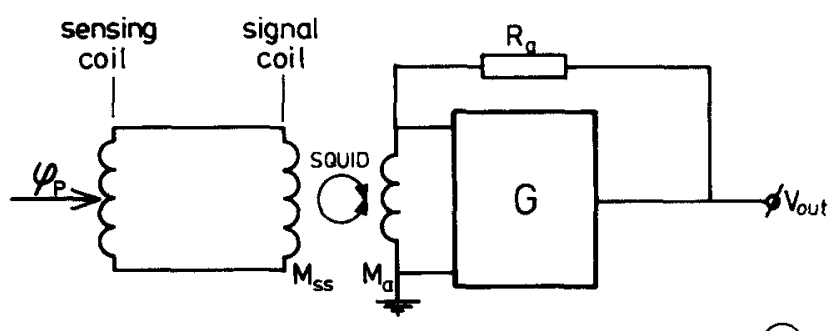

(a)

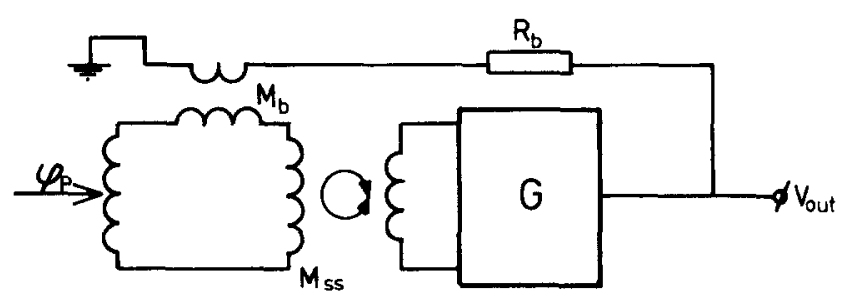

(b)

Figure 1 Schematic diagram of a SQUID system. (a) Internal feedback; (b) external feedback. $M_{s s}$, Mutual inductance between signal coil and SQUID; $G$, transfer function from SQUID flux to output voltage; $R_{\mathrm{a}, \mathrm{b}}$, feedback resistance; $M_{\mathrm{a}}$, mutual inductance between feedback loop and SQUID; $M_{\mathrm{b}}$, mutual inductance between feedback loop and flux-transformer circuit 
electronics is a direct measure for the magnetic flux coupled into the SQUID. To make the operation of the system linear, the output of the electronics is usually fed back to the SQUID so that the signal flux in the SQUID can be compensated (internal feedback, Figure 1a). In this case the flux-transformer carries a current, so crosstalk occurs in the multichannel system. This crosstalk can be eliminated by accommodating the feedback to the fluxtransformer circuit so that this circuit becomes currentless. This external feedback (Figure $1 b$ ) is accomplished by introducing a mutual inductor, of which the secondary coil is part of the flux-transformer circuit.

The transfer function from the signal flux in the sensing coil to the output voltage of the SQUID electronics (i.e. $V_{\text {out }} / \phi_{\mathrm{p}}$ ), with internal feedback (Figure $1 a$ ), is given by

$\left(V_{\text {out }} / \phi_{\mathrm{p}}\right)_{\text {int tb }}=R_{\mathrm{a}} M_{\mathrm{ss}} /\left[M_{\mathrm{a}}\left(L_{\mathrm{ft}}\right)_{\mathrm{a}}\right]$ for $G \gg R_{\mathrm{a}} / M_{\mathrm{a}}$

The current in the flux-transformer, $i_{\mathrm{ft}}$, is given by

$\left(i_{\mathrm{ft}}\right)_{\mathrm{int} \mathrm{fb}}=\phi_{\mathrm{p}} /\left(L_{\mathrm{ft}}\right)_{\mathrm{a}}$

In the case of external feedback (Figure $1 b$ ) the transfer function can be written as

$\left(V_{\text {out }} / \phi_{\mathrm{p}}\right)_{\text {ext fb }}=R_{\mathrm{b}} / M_{\mathrm{b}}$ for $G \gg R_{\mathrm{b}}\left(L_{\mathrm{ft}}\right)_{\mathrm{b}} /\left(M_{\mathrm{b}} M_{\mathrm{ss}}\right)$

and the flux-transformer current written as

$\left(i_{\mathrm{ft}}\right)_{\mathrm{ext} \mathrm{fb}}=\left[\phi_{\mathrm{p}} /\left(L_{\mathrm{ft}}\right)_{\mathrm{b}}\right]\left[1+G M_{\mathrm{b}} M_{\mathrm{ss}} /\left(R_{\mathrm{b}}\left(L_{\mathrm{ft}}\right)_{\mathrm{b}}\right)\right]^{-1}$

The self-inductance of the secondary coil of the mutual inductor in the external feedback loop adds to the self-inductance of the flux-transformer circuit. This results in a deterioration of the signal transfer from the sensing coil to the SQUID. Therefore, the inductance of the secondary coil has to be made small so that $\left(L_{\mathrm{ft}}\right)_{\mathrm{a}} \approx\left(L_{\mathrm{ft}}\right)_{\mathrm{b}}$. Equations (2) and (4) can now be used to evaluate the reduction of the flux-transformer current and thus the reduction of the crosstalk resulting from the rearrangement of the feedback loop. This reduction is found to be equal to the loop gain of the external feedback configuration.

\section{Testing configuration}

The crosstalk experiments were performed on a system of two second order gradiometer-type sensing coils (diameter $19.1 \mathrm{~mm}$, baseline length $40 \mathrm{~mm}$ ) each coil connected to a SHE 330 RF SQUID system (see Figure 2). The separate sections had three, six and three turns. The distance between the axes of the sensing coils was $25.5 \mathrm{~mm}$ (i.e. the smallest distance was $6.4 \mathrm{~mm}$ ). The self-inductance of each of the two sensing coils was matched to the input inductance of the SQUID device $(2 \mu \mathrm{H})$. The total self-inductance of a flux-transformer was, therefore, $\approx 4$ $\mu \mathrm{H}$. The mutual inductance between the sensing coils was calculated to be $47 \mathrm{nH}$, so that the crosstalk was $1.2 \%$.

A signal field was generated by means of two small input coils (six turns, diameter $3 \mathrm{~mm}$ ). These were positioned $11 \mathrm{~mm}$ below the sensing coils, as shown in Figure 2. The current through each of the input coils could be varied independently. Therefore, the flux which threaded sensing coil $B$, due to the input-coil currents, could be greatly reduced. The flux in coil B was, in this case, mainly caused by the crosstalk between both sensing coils. To distinguish the different effects, the crosstalk flux

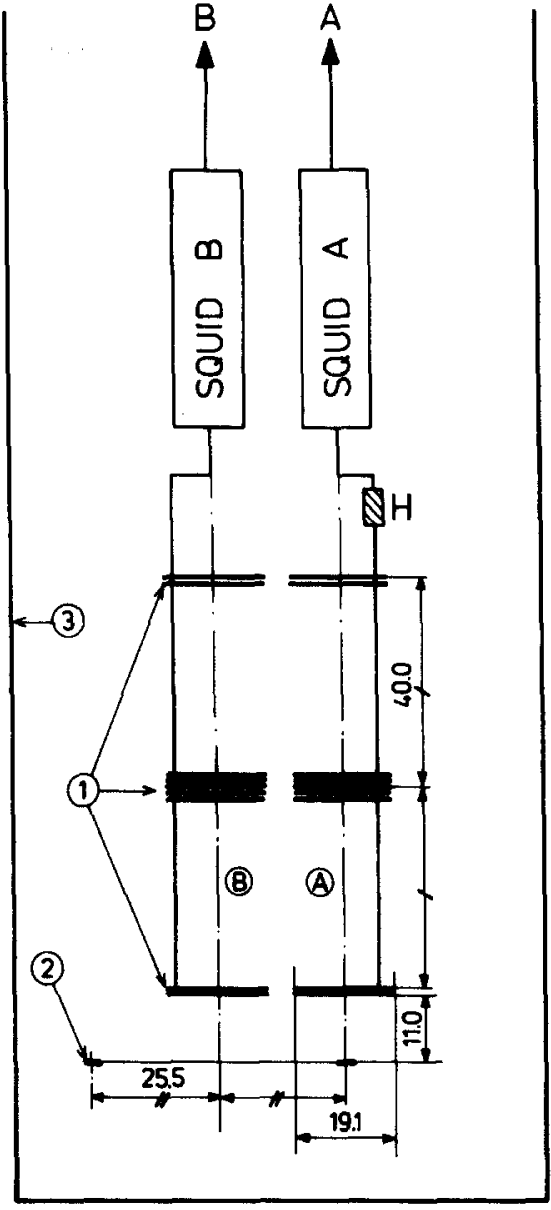

Figure 2 Schematic diagram of testing configuration (dimensions in $\mathrm{mm}$ ). 1, Sensing coil (second-order gradiometer) 2, input coil; 3, superconducting lead shield

could be eliminated by energizing the heater $H$ (see Figure 2).

System A could be operated in the internal as well as the external feedback mode, whereas system B could only be operated in the internal mode. The dimensions of the mutual inductor in the external feedback loop, given in Figure 3, are such that the inductor could be placed inside the superconducting niobium shield of the SQUID sensor. To reduce any coupling with the environment the inductor was constructed as an astatic coil set. The self-inductance of the primary coil (517 turns per section) was calculated

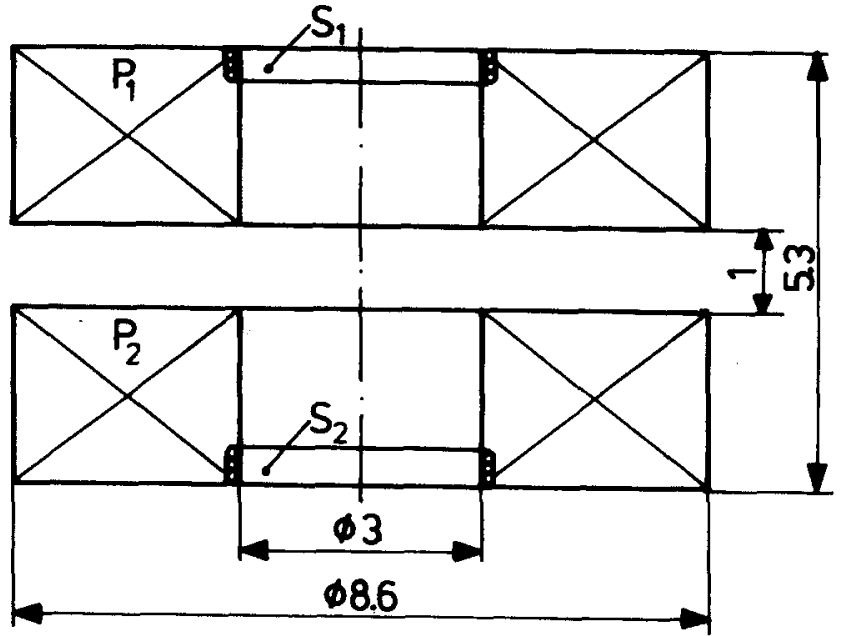

Figure 3 Feedback inductor (dimensions in $\mathrm{mm}$ ). $P_{1,2}$, primary coil-set; $S_{1,2}$, secondary coil-set 
to be $1.5 \mathrm{mH}$; the self-inductance of the secondary coil was found to be $0.1 \mu \mathrm{H}$ (three turns per section). The mutual inductance was $\approx 3.7 \mu \mathrm{H}$. The feedback resistance $\left(R_{\mathrm{b}}\right.$ in Figure $\left.1 b\right)$ was chosen to be $180 \mathrm{k} \Omega$. This value made the low frequency signal transfer from the sensing coil to the output of the SQUID electronics approximately the same for the external and internal feedback mode [see Equations (1) and (3)]. This signal transfer (sens $* 1$ mode) was $\approx 0.1 \mathrm{mV}$ per unit $\phi_{\mathrm{o}}\left(\phi_{\mathrm{o}}=2.07 \times 10^{-15} \mathrm{~Wb}\right)$.

To reduce RF interference the primary coil of the feedback inductor was shunted with a resistance of $1.5 \mathrm{k} \Omega$ (cut-off frequency $\approx 160 \mathrm{kHz}$ ). The effect of environmental noise was further suppressed by mounting the whole system inside a superconducting lead cylinder (see Figure 2).
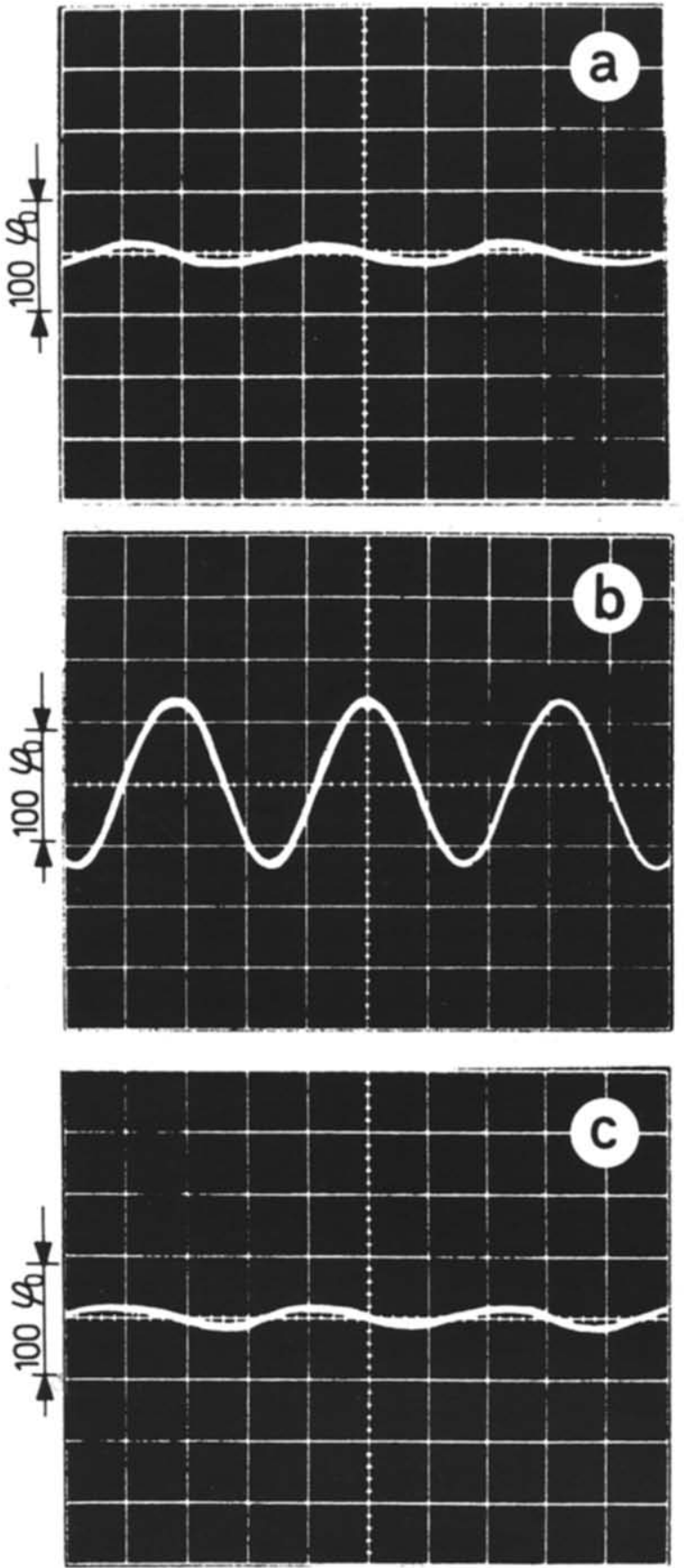

Figure 4 Suppression of flux-transformer crosstalk. (a) Flux in sensing coil $B$ with $A$ heated; (b) flux in sensing coil $B$ with $A$ not heated ( $A$ in internal feedback); (c) flux in sensing coil B with A not heated ( $A$ in external feedback). Vertical divisions $=\mathbf{5 3 . 8} \phi_{0}$; horizontal divisions $=10 \mathrm{~ms}$

\section{Results}

Figure 4 illustrates the successful suppression of fluxtransformer crosstalk by the use of external feedback. The experiments presented in this Paper were performed with a current of $\approx 4.2 \mathrm{~mA}_{\mathrm{RMS}}(30 \mathrm{~Hz}$ ) through the input coils. The currents were balanced so that the signal flux in coil $B$ was minimal with the heater in system $A$ energized (Figure 4a). A small flux in B is inevitable due to a minor phase shift between the individual input circuits. The peak-to-peak value of this flux was $\approx 15 \phi_{\mathrm{o}}\left(15 \phi_{\mathrm{opp}}\right)$. This corresponds to a phase shift of slightly more than one degree, with the flux due to each of the input coils $\approx$ $640 \phi_{\text {opp }}$. When system $A$ was operated in the internal feedback mode, a crosstalk flux $\approx 145 \phi_{\text {opp }}$ occurred in B as soon as the heating in A was stopped (Figure $4 b$ ). Because the signal flux in system A was $1.2 \times 10^{4} \phi_{\mathrm{opp}}$, the crosstalk $=1.2 \%$. This is in good agreement with the calculations mentioned above. However, when system A was operated in the external feedback mode no crosstalk flux occurred and the signal flux in B remained unaltered (15 $\phi_{\text {opp }}$ ) (Figure 4c).

It is, of course, important that the external feedback loop does not increase the noise level of the magnetometer. The spectral noise densities of system A, with
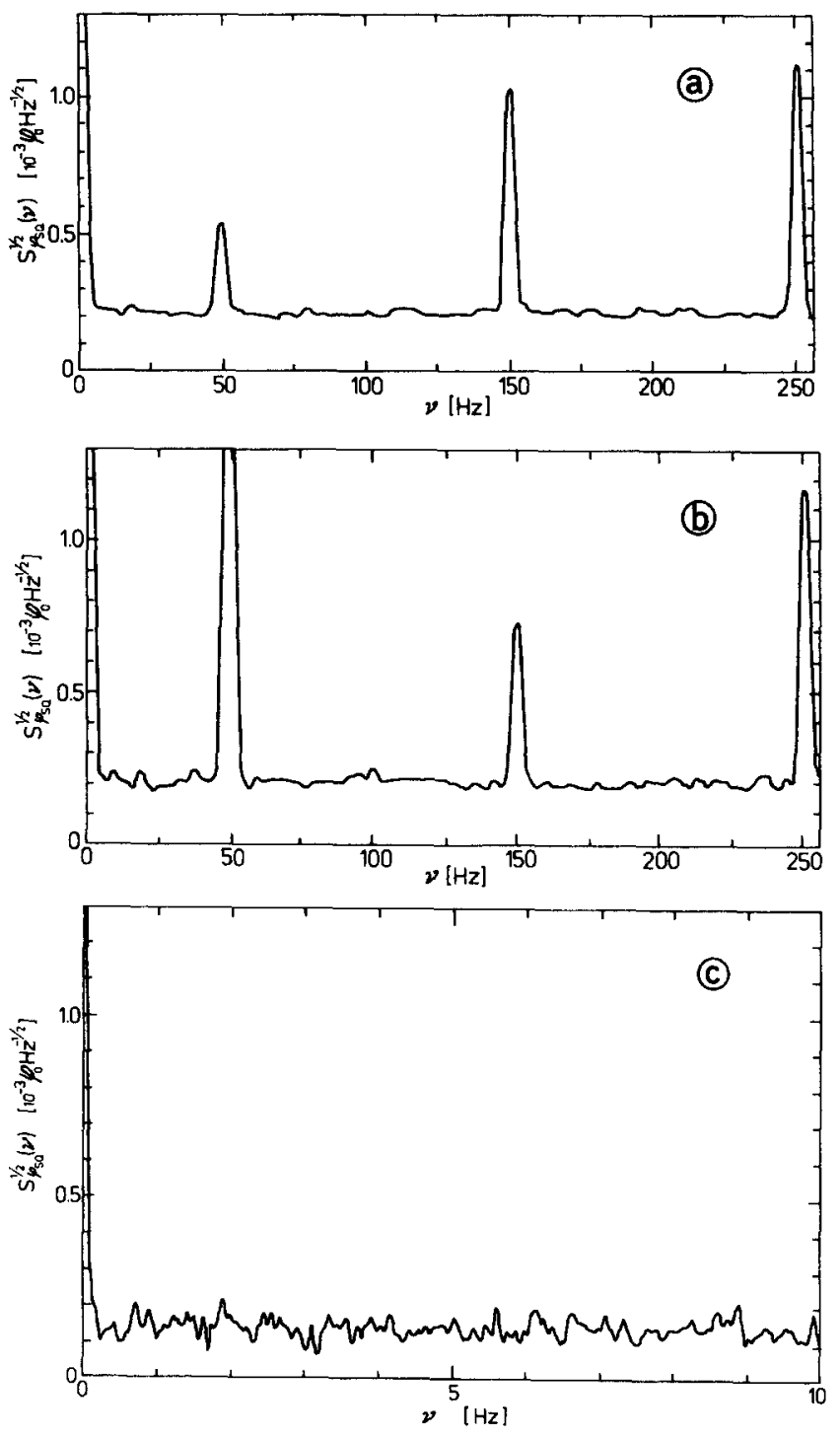

Figure 5 Spectral noise densities of system $A$ referred to the flux in the SOUID. (a) Internal feedback, $0-250 \mathrm{~Hz}$ (32 averages); (b) external feedback, $0-250 \mathrm{~Hz}$ (32 averages); (c) external feedback, $0-10 \mathrm{~Hz}$ (eight averages) 
internal and external feedback, were recorded by a spectrum analyser (HP $3582 \mathrm{~A}$ ). Typical recordings are shown in Figure 5. In this figure the noise levels are related to the flux in the SQUID. It appeared that the noise levels varied in time due to a variation in the $R F$ interference. In this respect the white-noise level for the internal feedback mode varied from $1 \times 10^{-4}$ to $4 \times 10^{-4}$ $\phi_{\mathrm{o}} \mathrm{Hz}^{-1 / 2}$. The typical white-noise level $\left(2 \times 10^{-4} \phi_{\mathrm{o}}\right.$ $\mathrm{Hz}^{-1 / 2}$ ) is presented in Figure $5 a$. This value is in agreement with the SHE specifications ${ }^{4}$. The noise spectrum (Figure $5 a$ ) shows peaks at 50,150 and $250 \mathrm{~Hz}$ due to power-line interference. The peak in the low frequency range is caused by the spectrum analyser.

When external feedback is applied (Figure $5 b$ ), the noise contribution of $50 \mathrm{~Hz}$ increases significantly. This is due to inadequate grounding in the external feedback circuit. The white-noise level in the external feedback mode is equivalent to that of the internal feedback mode. To examine the low frequency part of the spectrum, a recording was made in the region $0-10 \mathrm{~Hz}$ (Figure $5 c$ ). As this spectrum was recorded during a weekend (less RF interference), the white-noise level was relatively small $\left(\approx 1.5 \times 10^{-4} \phi_{0} \mathrm{~Hz}^{-1 / 2}\right)$. Using this spectrum we were able to confirm that the low frequency peaks in Figures $5 a$ and $b$ were indeed caused by the spectrum analyser. At $<$ $\approx 0.1 \mathrm{~Hz}$ the noise level of the system will be dominated by the $1 / f$ noise contribution of the SQUID system ${ }^{4}$.

\section{Conclusions}

We have demonstrated that the crosstalk between the individual sensing coils of a multichannel SQUID system can be substantially suppressed by means of an external feedback method. The incorporation of an additional mutual inductor in the flux-transformer circuit did not lead to an increase of the white-noise level of the system.

\section{References}

1 Erné, S.N. Proc Int Conf Superconducting Quantum Devices, SQUID '85 de Gruyter, West Berlin, FRG (1985) 891

2 Knuutila, J., Ahonen, A.I., Hămälăinen, M.S., Ilmoniemi, R.J. and Kajola, M.J. Proc Int Conf Superconducting Quantum Devices, SQUID '85 de Gruyter, West Berlin, FRG (1985) 939

3 Ilmoniemi, R.J., Hari, R. and Reinikainen, K. Electroencephalogr Clin Neurophysiol (1984) 58467

4 Specifications SHE 330 RF SQUID system 